\title{
Modeling and Designing Network Defense against Control Channel Jamming Attacks: A Passivity-Based Approach
}

\author{
Phillip Lee, Andrew Clark, Linda Bushnell, and Radha Poovendran \\ Dept. of Electrical Engineering, University of Washington, Seattle, WA, 98195, USA \\ \{leep3, awclark, lb2,rp3\}@uw.edu
}

\begin{abstract}
Cyber-physical systems rely on distributed embedded wireless nodes for sensing, computation, and control, often leaving them vulnerable to intelligent attacks. Detecting and mitigating such attacks in a resource-efficient manner requires the network to identify the components under attack and adapt its response based on the observed network performance and attack impact. In this paper, we present our ongoing work on a passivity-based, control-theoretic framework for designing and evaluating adaptive network defenses against the control channel jamming attack. In this attack, an adversary makes use of information gathered from the compromised nodes to identify the location and disrupt the control messages. We present a dynamical model of the attack impact over time, and represent the network response as a feedback control action. Using passivity theory, we identify a class of linear controllers that correspond to network responses that guarantee convergence to a desired operating point. Through a numerical study, we analyze network characteristics such as robustness to uncertain observations of the network state, stability in the presence of delayed observations, and rate of convergence to the desired state. We find that, in general, higherorder controllers provide increased robustness and stability, at the cost of slower overall convergence.
\end{abstract}

Keywords: Cyber-physical system, Node capture attacks, Security, Controlchannel jamming, Passivity

\section{Introduction}

Cyber-physical systems in a variety of settings are controlled by wireless networks that observe states of physical processes and transmit control signals in a distributed fashion [1]. When deployed in critical applications over wide geographic areas, these networks are susceptible to attacks due to their unattended nature. Moreover, the networks consist of resource-constrained low-cost embedded devices that can be physically captured and tampered by the adversary. These vulnerabilities can lead to a compromise of nodes [2] or external attacks that exploit the open wireless medium [3]. 
One such attack is the control-channel jamming attack where the adversary prevents nodes from receiving control packets by broadcasting an interfering signal during control packet transmission [4]. Control packets contain system parameters such as routing table update, or the access control messages and hence jamming these messages severely degrades network performance. Furthermore, since control messages are transmitted infrequently, and contained in short packets, control channel jamming can be performed by resource constrained mobile adversaries. The adversary can enhance the effectiveness of the control channel jamming attack by compromising valid nodes and extracting the location of control channels.

In order to maintain resilient network performance and functionality, defense mechanisms are needed to detect and remove compromised network nodes and update the control channels of valid nodes. Achieving high detection rate, however, can be costly in terms of network bandwidth, communications/storage overhead and energy consumption for resource constrained devices [5], creating a trade-off between resilience and cost of defense. Defense costs can be minimized by adaptively performing the detection and removal based on the observed system states, in order to drive the system to an operating point where the desired trade-off is achieved.

An adaptive defense against control channel jamming will be affected by several network properties. The effectiveness of defense mechanisms is impacted by delayed observations due to dropped packets. These observations may also be subjected to uncertainties in adversarial parameters such as the location and jamming power. Furthermore, different defense methods achieve varying speeds at which the network recovers to its desired state. An analytical approach is therefore required to compare and evaluate possible defense methods based on these criteria and determine which is most suitable for given network characteristics.

In this paper, we develop a control-theoretic framework for modeling and analyzing control channel jamming attacks and network defenses in cyber-physical systems. Under this framework, the attack and its impact are modeled as a dynamical system while the network response is represented by a feedback control action. We make the following contributions:

- We propose a passivity-based approach to design the network response based on the observed impact of attacks. We prove that the class of network responses corresponding to Strictly Positive Real (SPR) systems guarantee the convergence to a desired operating point, defined as the fraction of control messages that are correctly received.

- We show how to design SPR controllers to achieve and maintain a given operating point.

- We numerically evaluate a set of SPR controllers based on the criteria of (i) delay robustness, representing the robustness of the system to delayed observations due to packet drops, (ii) rejection of disturbances due to the uncertainties in the observations caused by unpredictable adversaries, and 
(iii) convergence rate, which corresponds to the speed of recovery of the system.

- We numerically show that the proportional controller achieves the higher convergence rate while the higher order controllers provide robustness to uncertainties. We also show that the delay robustness of the controller is determined by the performance at the steady state.

The paper is organized as follows. We review the related work in Section 2. The system model and background on passivity theory are given in Section 3. We present our control-theoretic framework in Section 4. Numerical results are presented in Section 5. Section 6 concludes the paper.

\section{Related Work}

Control channel jamming, in which the adversary broadcasts interference signal in order to disrupt control messages, has been identified as a threat to the functionality of wireless network. In [6], a channel assignment scheme was developed that enables the base station to determine the compromised nodes, up to a fixed threshold, based on the reported jammed channels. The channel assignment problem was mapped to key assignment problem in [4], providing non-zero detection probability even when a fraction of compromised nodes exceeds a given threshold. The channel assignment problem has also been studied using a coding theoretic framework in [7]. These mechanisms [4,6,7], however, do not model the progress of the attack over time. By providing such a temporal model, our approach enables the defense to change adaptively based on the observed impact of the attack, such as the reduced throughput of the control message or the fraction of jammed control channels.

A dynamical system model of the node capture/cloning attack in sensor networks was presented in [8]. The network response was derived as the solution of LQR/LQG problems. The derived network response, however, only utilizes the current observed impact of the attack and hence neglects previously observed information of the attack impact. In [9], a passivity framework was proposed which models the adversarial actions and the network response jointly. Sufficient conditions were derived that stabilize the network performance under different adversarial models. Design of specific control laws that satisfy the derived conditions, however, was not considered in [9].

\section{$3 \quad$ Model and Preliminaries}

In this section, we describe our models for the networked system, adversary, and system defense. In particular, we describe how the adversary can increase the impact of control jamming attack by jointly performing node capture attack. We also give relevant background on passive systems. 


\subsection{Networked System Model}

We consider a system of $n$ nodes, which communicate via a wireless network using a time-slotted communication protocol such as orthogonal frequency-division multiple access (OFDMA). The network is facilitated by a base station, which sends necessary information for network functionality, such as scheduling data and public encryption keys, in control messages. Control messages are sent via dedicated control channels. In order to provide such channels, we assume that, periodically, the initial portion of a time slot is dedicated to control messages, while the remainder of the slot is used for data transmission. The portion of the slot dedicated to control messages is further divided into $S$ sub-slots. During each sub-slot, control messages can be transmitted on one or more frequency bands, out of $M$ available frequencies. A (sub-slot, band) pairing defines a control channel (Figure 1).

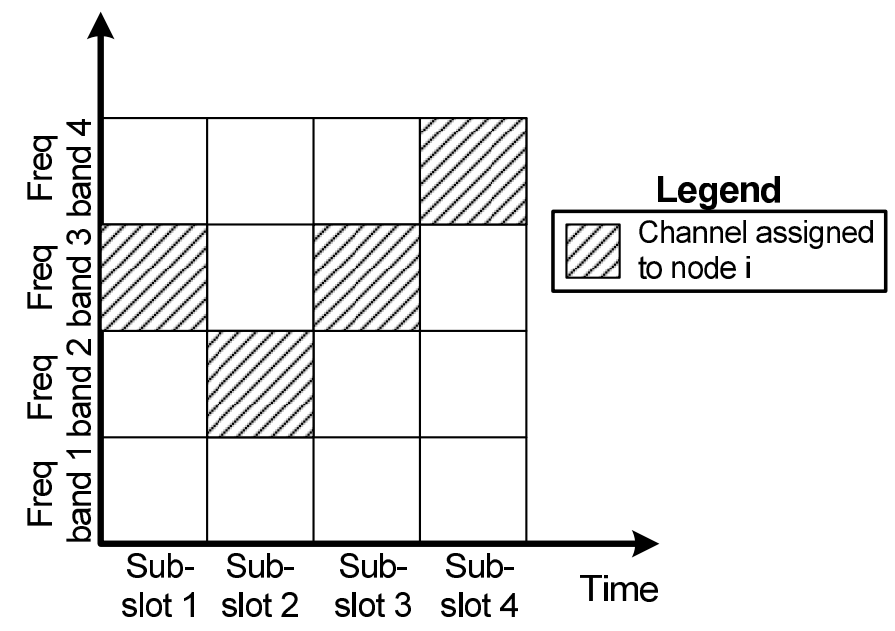

Fig. 1. Illustration of control channel assignment. Each (sub-slot, band) pairing defines a control channel. Node $i$ is assigned the channels (sub-slot 1, band 3), (sub-slot 2, band 2), (sub-slot 3, 3), (sub-slot 4, band 4)

In order to prevent jamming of control messages, the control channel used by each node varies between time slots. Letting $c_{i j}$ denote the control channel of node $i$ during time slot $j$, the channel hopping sequence of node $i$ is given by $\mathcal{C}_{i}=\left\{c_{i 1}, c_{i 2}, \ldots,\right\}$. We assume that $\mathcal{C}_{i}$ repeats itself with period $p$, so that the sequence $i$ is uniquely defined by the period $\overline{\mathcal{S}}_{i}=\left\{c_{i 1}, \ldots, c_{i p}\right\}$. In order to determine $c_{i j}$, each node is assumed to have access to a secret seed $K_{i}$, known only to node $i$ and the base station. Node $i$ computes $c_{i j}$ as $h\left(K_{i}, j\right)$, where $h(\cdot): \mathcal{K} \times \mathbb{Z} \rightarrow \mathcal{M}$ is a pseudorandom function, $j=t \bmod p, \mathcal{M}$ is the set of available frequencies, and $\mathcal{K}$ is the set of possible seeds [4]. To reduce the 
overhead at the base station, the seeds $\left\{K_{i}: i=1, \ldots, n\right\}$ may be chosen so that the hopping sequences overlap, i.e., $\mathcal{C}_{i} \cap \mathcal{C}_{j} \neq \emptyset$, reducing the number of times that the base station must retransmit each control message.

\subsection{Adversary Model}

The network operates in the presence of one or more mobile, intelligent adversaries, whose goal is to prevent a subset of victim nodes from receiving control messages. In order to obtain the hopping sequences used by the base station, the adversary compromises a set of nodes, denoted $\mathcal{A}$, and thereby gains access to the seeds $\mathcal{K}_{\mathcal{A}}=\left\{K_{i}: i \in \mathcal{A}\right\}$. After capturing a seed $K_{i}$, the adversary computes the publicly available function $h\left(K_{i}, j\right)$ at time slot $j$, and can then jam all control channels in the sequence $\mathcal{C}_{i}$. Due to the overlap between channel hopping sequences of different nodes, a jamming attack on the channels $\mathcal{C}_{i}$ may prevent other, valid nodes from receiving control messages.

The base station and nodes detect and respond to the attack as follows. Each valid node is assumed to have a secure feedback channel to the base station for reporting errors in received control messages. Based on this feedback received from valid nodes, the base station identifies a set of control channels $\hat{\mathcal{C}}$ that may be under jamming attack. Since a control channel can be jammed when one of the corresponding seeds is compromised, the set of compromised nodes is then estimated based on the set $\hat{\mathcal{C}}$. In particular, the base station identifies a set of nodes $\hat{\mathcal{A}}$ such that $\hat{\mathcal{C}} \subseteq \mathcal{K}_{\hat{\mathcal{A}}}$. Nodes that are suspected of being compromised are removed from the network, while any node $i$ satisfying $\mathcal{C}_{i} \cap \mathcal{C}_{j} \neq \emptyset$ for some compromised node $j$ is issued a new seed $K_{i}^{\prime}$.

\subsection{Background on Passivity}

In what follows, we consider a state-space model $(\Sigma)$, with state $x(t)$, input $u(t)$, and output $y(t)$, defined by

$$
(\Sigma)\left\{\begin{array}{l}
\dot{x}(t)=f(x(t), u(t)) \\
y(t)=g(x(t), u(t))
\end{array}\right.
$$

The definitions and results in this subsection can be found in [10]. A passive system is defined as follows.

Definition 1. The system $(\Sigma)$ is passive iff there exists a nonnegative $\mathcal{C}^{1}$ function $V: \mathbb{R} \rightarrow \mathbb{R}_{\geq 0}$ satisfying $V(0)=0$ and

$$
\dot{V}(t) \leq-S(x(t))+u(t)^{T} y(t)
$$

where $S(\cdot)$ is a nonnegative continuous function. If $S(x)>0$ for all $x \neq 0$, then the system is strictly passive. A function $V$ satisfying (1) for a system $(\Sigma)$ is a storage function for $(\Sigma)$.

A passive system response should be efficiently computable by the nodes and base station. One such class of passive systems consists of linear systems that satisfy the strict positive realness $(S P R)$ property, defined below. 
Definition 2. Consider the linear system $\left(\Sigma_{L}\right)$, defined by

$$
\left(\Sigma_{L}\right)\left\{\begin{array}{l}
\dot{x}(t)=A x(t)+B u(t) \\
y(t)=C x(t)+D u(t)
\end{array}\right.
$$

Then $\left(\Sigma_{L}\right)$ is positive real if its transfer function $h(s)$ is real for positive real $s$ and satisfies $\mathbf{R e}[h(s)] \geq 0$ for all $\mathbf{R e}[s]>0 . \Sigma_{L}$ is strictly positive real if $h(s-\epsilon)$ is positive real for some $\epsilon>0$.

A linear system is passive if and only if it is positive real, and is strictly passive if and only if it is strictly positive real. The Kalman-Yakubovich-Popov lemma gives equivalent conditions for a linear system to be SPR.

Lemma 1 (KYP). The transfer function of $\left(\Sigma_{L}\right)$ is strictly positive real if and only if there exist matrices $P=P^{T}>0, L, W$, and a positive constant $\epsilon>0$ such that

$$
\begin{aligned}
P A+A^{T} P & =-L L^{T}-\epsilon P \\
P B-C^{T} & =-L W \\
D+D^{T} & =W^{T} W
\end{aligned}
$$

Passivity leads to a variety of techniques for guaranteeing stability of dynamical systems, such as the following proposition.

Proposition 1. A negative feedback interconnection between two strictly passive systems is globally asymptotically stable.

In particular, a negative feedback interconnection between a strictly passive system and an SPR system is globally asymptotically stable. For a negative feedback interaction between two strictly passive systems with storage functions $V_{1}$ and $V_{2}$, the function $V=V_{1}+V_{2}$ is a Lyapunov function for the combined system.

\section{Control-Theoretic Framework for Mitigating Control Channel Jamming}

In this section, we present a control-theoretic framework for modeling and mitigating control-channel jamming attacks, in which the attack is represented as a nonlinear dynamical system and the network response is the feedback control. We analyze the steady-state behavior and prove that the class of linear SPR controllers leads to network responses that converge to a desired steady-state value, which can be tuned by choosing the parameters of the controller.

\subsection{Dynamical Model of Attack and Network Response}

Our dynamical modeling approach consists of two components, namely, a model of the attack dynamics and a model of the system's defense mechanisms. For 
the attack dynamics, we consider an affine model first introduced in [9]. Let $x(t)$ denote the fraction of nodes that have been compromised at time $t$. Define $f:[0,1] \rightarrow \mathbb{R}_{\geq 0}$ to be a nonnegative, continuous, and increasing function, such that $f(x)$ quantifies the impact of the attack on the system performance when the fraction of compromised nodes is $x$. The dynamical model of the attack is given by

$$
\left(\Sigma_{\text {sys }}\right)\left\{\begin{aligned}
\dot{x}(t) & =\lambda(1-x(t))+u_{1}(t) \\
y_{1}(t) & =f(x(t))
\end{aligned}\right.
$$

where $\lambda$ represents the rate at which nodes are captured. The model (2) reflects the fact that, as the adversary compromises nodes, the incremental effort to locate and compromise an additional node increases, thus reducing the capture rate.

The network response is defined by the rate at which compromised nodes are detected and revoked and removed from the system, denoted $y_{2}(t)$, so that $\dot{x}(t)=\lambda(1-x(t))-y_{2}(t)$. We consider a revocation rate $y_{2}(t)$ that is given as the output of a linear system of the form

$$
\left(\Sigma_{\text {control }}\right)\left\{\begin{aligned}
\dot{\xi}(t) & =A \xi(t)+B f(x) \\
y_{2}(t) & =C \xi(t)+D f(x)
\end{aligned}\right.
$$

The $D f(x)$ term represents the network response to the currently-observed impact of the attack, $f(x)$. The state variable $\xi(t)$ is based on the attack dynamics from $[0, t]$, and hence represents the past observations. Tuning the parameters $(A, B, C, D)$ therefore determines how rapidly the system will respond to changes in network performance caused by the attack.

Based on (2) and (3), the combined system consisting of the control channel jamming attack and network response is interpreted as a negative feedback interconnection, where the network response acts as a feedback control, as illustrated in Figure 2. In what follows, we first derive the steady-state operating point

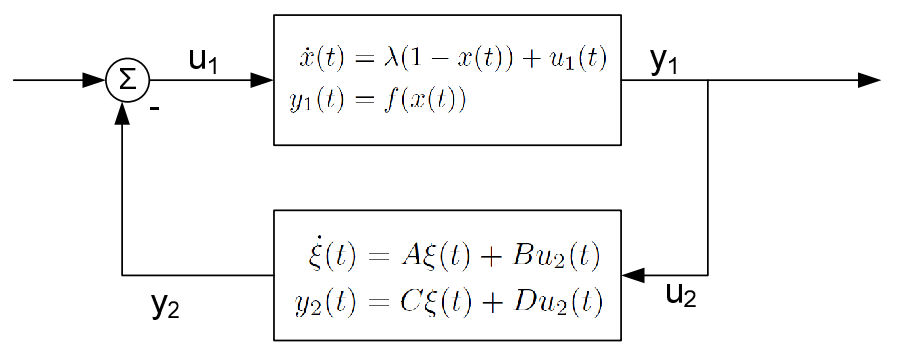

Fig. 2. Attack and response dynamics.

$\left(x^{*}, \xi^{*}\right)$ of the system in Figure 2. The steady-state $x^{*}$ represents the fraction of nodes that are compromised, and hence the fraction of channels that are jammed 
$f\left(x^{*}\right)$, after the system has been deployed for a sufficiently long time period. We show that an arbitrary value of $x^{*} \in(0,1)$ can be achieved by selecting the matrices $(A, B, C, D)$. We then prove that, for the class of strictly positive real systems $\Sigma_{\text {control }}$, the combined system is globally asymptotically stable.

\subsection{Steady-state Value}

The desired steady-state of the system can be determined by trading off the impact of the attack on the network performance with the resource cost of mitigating the attack [5]. Once a desired steady-state $x^{*}$ has been chosen, the goal is to design a control law defined by $\Sigma_{\text {control }}$ that achieves this value.

The steady-state is derived of (2) and (3) is derived as follows. $\dot{\xi}(t)$ is equal to zero when $\xi=\xi^{*}=-A^{-1} B f\left(x^{*}\right)$. Substituting into (2) and setting $\dot{x}(t)=0$ yields

$$
\lambda\left(1-f^{-1}\left(y^{*}\right)\right)+\left(C A^{-1} B-D\right) y^{*}=0,
$$

where we use the fact that $f$ is continuous and increasing, and hence invertible. Set $r=\lambda\left(1-f^{-1}\left(y^{*}\right)\right)$ and $\psi=\left(C A^{-1} B-D\right)$. Then $y^{*}$ is the steady-state if $\psi=-r / y^{*}$, i.e., if $C A^{-1} B-D=-r / y^{*}$. This can be accomplished in different ways, such as selecting $D$ in order to ensure that the condition is satisfied, or by scaling the matrices $(A, B, C, D)$ by the same value in order to achieve the desired ratio.

\subsection{Global Asymptotic Stability}

In order to prove global asymptotic stability of (2) and (3), we first introduce new variables representing the deviation of the state from the steady-state value, denoted as $\hat{x} \triangleq x-x^{*}, \hat{\xi} \triangleq \xi-\xi^{*}$. Rewriting the dynamics in terms of $\hat{x}$ and $\hat{\xi}$ yields

$$
\begin{aligned}
\dot{\hat{x}}(t)= & \dot{x}(t)=\lambda\left(1-\hat{x}(t)-x^{*}\right)-C\left(\hat{\xi}(t)+\xi^{*}\right) \\
& -D\left(f\left(\hat{x}(t)+x^{*}\right)-f\left(x^{*}\right)+f\left(x^{*}\right)\right) \\
= & -\lambda \hat{x}(t)-C \hat{\xi}(t)-D\left(f\left(\hat{x}(t)+x^{*}\right)-f\left(x^{*}\right)\right) \\
\dot{\hat{\xi}}(t)= & \dot{\xi}(t)=A\left(\hat{\xi}(t)+\xi^{*}\right)+B\left(f\left(\hat{x}(t)+x^{*}\right)-f\left(x^{*}\right)+f\left(x^{*}\right)\right) \\
= & A \hat{\xi}(t)+B\left(f\left(\hat{x}(t)+x^{*}\right)-f\left(x^{*}\right)\right) .
\end{aligned}
$$

Here, (4) follows from the fact that, since $\left(x^{*}, \xi^{*}\right)$ is a steady-state value for (2) and (3), $\lambda\left(1-x^{*}\right)-C \xi^{*}-D f\left(x^{*}\right)=0$. Similarly, (5) follows from the fact that $A \xi^{*}+B f\left(x^{*}\right)=0$.

The equivalent dynamics (4) and (5) are described by the block diagram in Figure 3. In essence, the dynamics are equivalent to a negative feedback interconnection between a system $H_{1}$, defined by

$$
\left(H_{1}\right)\left\{\begin{array}{c}
\dot{\hat{x}}(t)=-\lambda \hat{x}(t)+u_{1}(t) \\
y_{1}(t)=f\left(\hat{x}+x^{*}\right)-f\left(x^{*}\right)
\end{array}\right.
$$




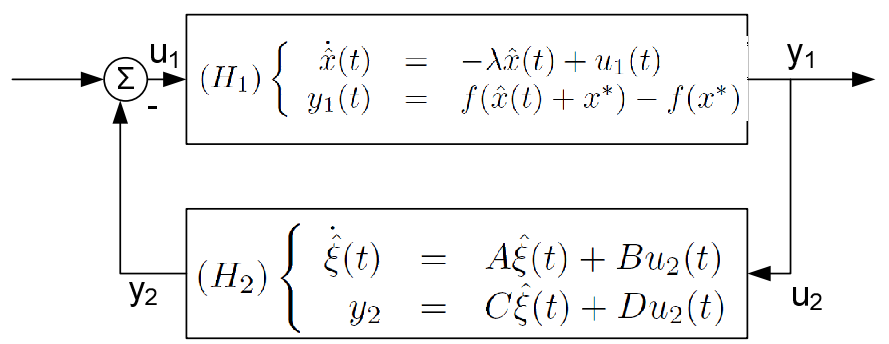

Fig. 3. Equivalent representation as negative feedback interconnection between two passive systems.

and a system $H_{2}$ defined by

$$
\left(H_{2}\right)\left\{\begin{aligned}
\dot{\hat{\xi}}(t) & =A \hat{\xi}(t)+B u_{2}(t) \\
y_{2}(t) & =C \hat{\xi}(t)+D u_{2}(t)
\end{aligned}\right.
$$

The following lemma concerns the system $H_{1}$.

Lemma 2. The system $H_{1}$ is passive from the input $u_{1}$ to the output $y_{1}$.

Proof. In order to prove passivity, the goal is to find a function $V(\hat{x})$ such that $V(0)=0, V(\hat{x}) \geq 0$ for all $\hat{x} \in \mathbb{R}$, and $\dot{V}(\hat{x}(t)) \leq u_{1}(t) y_{1}(t)$. The candidate function is given by

$$
V(\hat{x})=\int_{x^{*}}^{\hat{x}+x^{*}} f(a) d a-\hat{x} f\left(x^{*}\right)
$$

First, note that $V(0)=0$. To see that $V(\hat{x}) \geq 0$, first consider the case that $\hat{x}>0$. Since $V(0)=0$, it suffices to show that $\frac{\partial V}{\partial \hat{x}} \geq 0$ for $\hat{x}>0$. In this case, $\frac{\partial V}{\partial \hat{x}}=f\left(\hat{x}+x^{*}\right)-f\left(x^{*}\right) \geq 0$, where the inequality holds because $\hat{x}>0$ and $f$ is increasing.

Now, when $\hat{x}<0$, we write $\hat{x}=-s$ for some $s>0$. Thus $V(\hat{x})$ is given by

$$
\begin{aligned}
V(\hat{x}) & =\int_{x^{*}}^{x^{*}-s} f(a) d a+s f\left(x^{*}\right)=-\int_{x^{*}-s}^{x^{*}} f(a) d a+s f\left(x^{*}\right) \\
& =-\int_{x^{*}-s}^{x^{*}} f(a) d a+\int_{x^{*}-s}^{x^{*}} f\left(x^{*}\right) d a=\int_{x^{*}-s}^{x^{*}} f\left(x^{*}\right)-f(a) d a .
\end{aligned}
$$

Since $f$ is increasing, $f\left(x^{*}\right) \geq f(a)$ for all $a \leq x^{*}$. Hence $V(\hat{x})$ is the integral of a nonnegative function, and is therefore nonnegative.

Finally, we want to show that $\dot{V}(\hat{x}(t)) \leq u_{1}(t) y_{1}(t)$ for all $\hat{x}(t) \in \mathbb{R}$. We have

$$
\begin{aligned}
\dot{V}(\hat{x}(t)) & =\left(f\left(\hat{x}(t)+x^{*}\right)-f\left(x^{*}\right)\right)\left(-\lambda \hat{x}(t)+u_{1}(t)\right) \\
& =-\lambda\left(f\left(\hat{x}(t)+x^{*}\right)-f\left(x^{*}\right)\right) \hat{x}(t)+u_{1}(t) y_{1}(t)
\end{aligned}
$$


If $\hat{x}(t)>0$, then $f\left(\hat{x}(t)+x^{*}\right)>f\left(x^{*}\right)$, and hence the first term of (6) is negative. If $\hat{x}(t)<0$, then $f\left(\hat{x}(t)+x^{*}\right)<f\left(x^{*}\right)$, and the first term of (6) is therefore negative. Thus $\dot{V}(\hat{x}(t)) \leq u_{1}(t) y_{1}(t)$ for all $\hat{x}(t) \in \mathbb{R}$, which establishes the passivity of $H_{1}$.

Theorem 1. Suppose that the matrices $(A, B, C, D)$ in $\mathrm{H}_{2}$ define a strictly positive real transfer function. Then the dynamics of (2) and (3) are globally asymptotically stable with steady state $\left(x^{*}, \xi^{*}\right)$.

Proof. If $(A, B, C, D)$ define a strictly positive real system, then $H_{2}$ is passive, and hence the joint dynamics (2) and (3) are equivalent to a negative feedback interconnection between two strictly passive systems, and is therefore passive.

\subsection{Ensuring Correct Values of $x(t)$}

The last remaining task is to ensure that the dynamics of $x(t)$ does not leave the feasible region $x(t) \in[0,1]$. This is accomplished by using the following modified control law

$$
\left(\Sigma_{\text {mod }}\right)\left\{\begin{array}{c}
\dot{x}(t)=\lambda(1-x(t))-C \xi(t) \mathbb{1}(\mathcal{R})-D f(x) \\
\dot{\xi}(t)=A \xi(t)+B f(x)
\end{array}\right.
$$

where $\mathbb{1}(\cdot)$ denotes the indicator function and

$$
\mathcal{R} \triangleq\{f(x) \in(0, f(1))\} \cup\{f(x) \geq f(1), C \xi>0\} \cup\{f(x) \leq 0, C \hat{<} 0\},
$$

noting that the control action described can be implemented with knowledge only of $f(x)$. We now show that the modified dynamics of $\Sigma_{\text {mod }}$ satisfy $x(t) \in[0,1]$ $\forall t \geq 0$ and global asymptotic stability of the same equilibrium point $\left(x^{*}, \xi^{*}\right)$.

Proposition 2. The dynamics of $\Sigma_{\text {mod }}$ satisfy $x(t) \in[0,1]$, provided that $(A, B, C, D)$ is strictly positive real, $f$ is increasing, and $x(0) \in[0,1]$.

Proof. Since the dynamics is continuous, it suffices to show that $\dot{x}(t)>0$ when $x(t)=0$ and $\dot{x}(t)<0$ when $x(t)=1$. If $x(t)=0$, we have

$$
\dot{x}(t)=\lambda-C \xi(t) \mathbb{1}(\mathcal{R})
$$

since $f(0)=0$. Now, $C \xi(t)>0$, then $\mathbb{1}(\mathcal{R})=0$. Hence $\dot{x}(t) \geq \lambda>0$, as desired. Similarly, if $x(t)=1$, then

$$
\dot{x}(t)=-C \xi(t) \mathbb{1}(\mathcal{R})-D f(1) .
$$

If $C \xi(t)<0$, then $\mathbb{1}(\mathcal{R})=0$, and hence $\dot{x}(t) \leq-D f(1)$. Since $(A, B, C, D)$ is strictly positive real, the KYP lemma implies that $D>0$, and so $D f(1)>0$. Thus $\dot{x}(t) \leq-D f(1)<0$ when $x(t)=1$.

It remains to show that the global asymptotic stability still holds for the modified dynamics $\Sigma_{\text {mod }}$. This result is provided by the following proposition. 
Proposition 3. The dynamics of $\Sigma_{m o d}$ are globally asymptotically stable with equilibrium point $\left(x^{*}, \xi^{*}\right)$.

Proof. As in Lemma 2 and Theorem 1, we consider the stability of the system defined by $\hat{x}(t)=x(t)-x^{*}$ and $\hat{\xi}(t)=\xi(t)-\xi^{*}$. In order to prove global asymptotic stability, it suffices to find a Lyapunov function $V(x(t), \xi(t))$ such that $\frac{d V}{d t}<0$. Since the $H_{1}$ and $H_{2}$ of Figure 3 are passive, the function $V(\hat{x}(t), \hat{\xi}(t))=V_{1}(\hat{x}(t))+V_{2}(\hat{\xi}(t))$, where $V_{1}$ and $V_{2}$ are storage functions for $H_{1}$ and $H_{2}$, respectively, is a Lyapunov function for the system in Figure 3 . We prove that $V(\hat{x}(t), \hat{\xi}(t))$ is also a Lyapunov function for the system defined by $\Sigma_{\text {mod }}$.

For the system defined by $\Sigma_{\text {mod }}$, we have

$$
\begin{aligned}
\dot{V}(\hat{x}(t), \hat{\xi}(t)) & =\frac{\partial V}{\partial \hat{x}} \dot{\hat{x}}(t)+\frac{\partial V}{\partial \xi} \dot{\xi}(t) \\
& =\left(f(x(t))-f\left(x^{*}\right)\right)(\lambda(1-x(t))-[C \xi(t)] \mathbb{1}(\mathcal{R})-D f(x))+\frac{\partial V}{\partial \xi} \dot{\xi}(t) \\
& \leq\left(f(x(t))-f\left(x^{*}\right)\right)(\lambda(1-x(t))-C \xi(t)-D f(x))+\frac{\partial V}{\partial \xi} \dot{\xi}(t)<0
\end{aligned}
$$

where the final inequality follows from the global asymptotic stability of the system in Figure 3. Hence $V(\hat{x}(t), \hat{\xi}(t))$ is a Lyapunov function for $\Sigma_{\text {mod }}$, implying that the system is globally asymptotically stable.

\section{Numerical Study}

We conduct a numerical study using Matlab. The goal of this study is to evaluate different SPR controllers using the metrics 1) convergence rate, 2) disturbance rejection in the observation errors, and 3) robustness to delay in observation.

We consider the capture rate $\lambda=0.1$. The impact function $f(x)$ has been linearized around the $x^{*}$, and assume that $f(x) \approx x$. The initial condition $x(0)=0$. Our results for each of the metrics are as follows.

\subsection{Robustness to Imperfect Observation}

Given different network responses, we first compare the ability of the network to reach the desired operating state when observations $f(x)$ are imperfect. Imperfect observations may be due to uncertainty in the adversarial model or noisy communication channels. Robustness to imperfect observations is simulated by comparing the stability performance between the proportional controller and the higher order SPR controller in the presence of additive noise $\omega(t)$ introduced in the observation. The simulated noise is white zero mean Gaussian with variance of 4. Proportional controller has parameter values $A=B=C=0$, and $D=0.4$. The higher order SPR controller has parameter values $A=-\frac{1}{64}, B=\frac{1}{16}, C=\frac{1}{10}$ and $D=0$. The steady state value is $x^{*}=0.2$ in both cases. The average stability performance is estimated via Monte-Carlo methods. We performed 300 

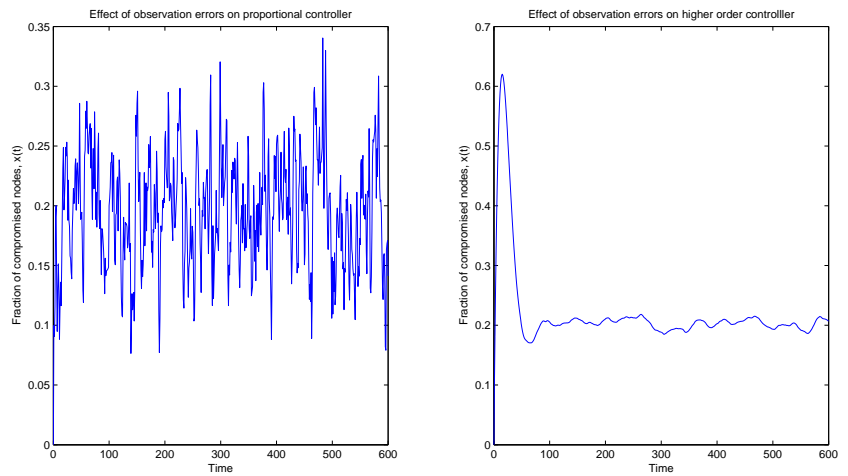

Fig. 4. Simulation results comparing the stability performance given additive white Gaussian noise in the observation. Gaussian noise had statistical property of zero mean and variance 4. $x^{*}=0.2$, which resulted in $D=0.4$ for the proportional controller (left plot). For the higher order controller, $A=-\frac{1}{64}, B=\frac{1}{16}, C=\frac{1}{10}$ values were found heuristically in order to achieve the same $x^{*}=0.2$. The higher order controller provides superior noise rejection at the steady state at the cost of higher attack impact prior to convergence.

trials for both controllers with independent noise. The results are illustrated in Figure 4. The higher order controller is more robust to observation errors than the proportional controller. In the proportional case, $D$ is determined uniquely by $x^{*}$. Since the impact of observation error introduced in the state dynamics is additive error of $D \omega(t)$, the impact of additive error cannot be decreased without changing the steady state value $x^{*}$. On the other hand, in the higher order SPR controller, $x^{*}=\frac{1}{1-\frac{1}{\lambda} \frac{B C}{A}}$. This enables higher degree of freedom in choosing $A, B, C$ values to achieve $x^{*}$ while minimizing the impact of observation error.

\subsection{Robustness to Delay in Observation}

Given different network responses, we next compare the ability of the network to reach the desired operating state when observations are delayed due to packet drops. Robustness to delay is simulated by comparing the stability performance between the proportional controller and the higher order SPR controller in the presence of delay in observation. A delay of 5 time units was introduced in the observation data. The proportional controller has parameter values $A=B=$ $C=0$, and $D=0.4$. The higher order SPR controller has parameter values $A=-\frac{1}{64}, B=\frac{1}{16}, C=\frac{1}{10}$ and $D=0$. The steady state value is $x^{*}=0.2$ in both cases. The results are illustrated in Figure 5. Given fixed delay and steady state value $x^{*}$, the higher order SPR controller enables convergence to the desired steady state value, while convergence to the steady state value can no longer be guaranteed in the proportional controller case. Since $D$ is uniquely determined by $x^{*}$ in the proportional controller case, the simulation result proves that there 

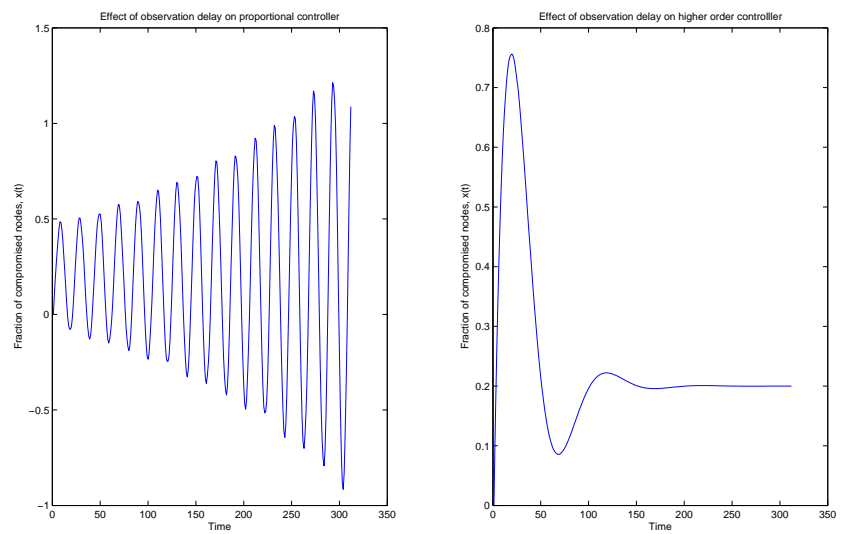

Fig. 5. Simulation results comparing the robustness to delay in observation. A delay of 5 time units was introduced in the observation data. The steady-state value is $x^{*}=0.2$, which resulted in $D=0.4$ for the proportional controller (left plot). For the higher order controller, $A=-\frac{1}{64}, B=\frac{1}{16}, C=\frac{1}{10}$ values were found heuristically in order to achieve the same $x^{*}=0.2$. The higher order controller provides superior performance in satisfying convergence to $x^{*}$ in the presence of delay.

exists a set of higher order SPR controllers which provide robustness to delay given a fixed $x^{*}$. It should be noted the higher order SPR controller with the parameters given above is quite robust to delay. Simulation results, which are omitted here, suggest that the higher order controller guarantee convergence to the steady state of 0.2 up to approximately 20 units of time delay in observation.

\subsection{Convergence Rate}

We compare how fast the network reaches the desired fraction of compromised nodes for given network responses. In the case of no noise in the observation or delay, we compare the convergence rate of the higher order controller with

same parameter values given above $\left(A=-\frac{1}{64}, B=\frac{1}{16}, C=\frac{1}{10}, D=0\right)$ to the proportional controller with $D=0.4$. The results are illustrated in Figure 6 . The same higher order controller that provided robustness to delay and noisy observation has a much slower convergence rate than the proportional controller. The higher order SPR controller utilizes the past observations and hence is slower to respond to current observation. This suggests that there is a trade-off between the convergence rate and the robustness to delayed and noisy observations.

\section{Conclusion}

In this paper, we presented a control-theoretic framework for modeling and analyzing defenses against attacks on cyber-physical systems. We considered the 

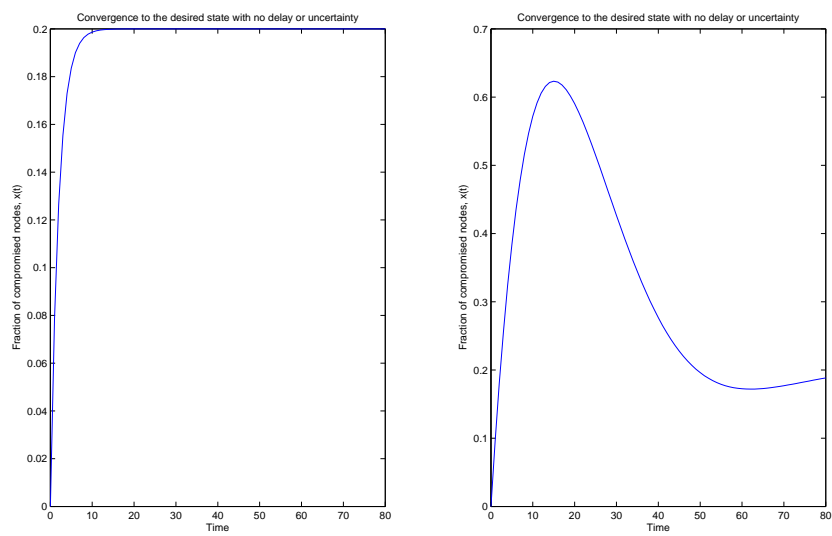

Fig. 6. Simulation results comparing the convergence rate. $x^{*}=0.2$, which resulted in $D=0.4$ for the proportional controller (left plot). For the higher order controller, $A=-\frac{1}{64}, B=\frac{1}{16}, C=\frac{1}{10}$ values were found heuristically in order to achieve the same $x^{*}=0.2$. The proportional controller achieves steady state within 15 time units while the higher order SPR controller does not achieve the steady state within 80 time units.

control channel jamming attack, in which an adversary compromises a set of nodes and uses information extracted from the nodes to jam control messages sent from a base station. The attack dynamics and impact were modeled as a dynamical system, while the network response was represented as a feedback control action. Using a passivity-based approach, we identified a class of response dynamics, corresponding to the set of strictly positive real controllers, that guarantee convergence to a steady-state value, and proved that a controller can be chosen to achieve any steady state. We further showed that different control laws within this space result in different network performance characteristics, including robustness to delays and observation errors, as well as rate of network recovery from the attack. In particular, through a numerical study we found that choosing a detection and removal rate that is proportional to the fraction of jammed channels leads to faster recovery, but is less robust to delays and uncertainties than a higher-order control law. Analytically verifying these results by finding the maximum observation delay that can be tolerated while maintaining stability, as well as deriving bounds on the convergence rate and noise robustness, will be the subject of future work. Moreover, we will study a general set of attack dynamics such as replicator-type dynamics while incorporating the multi-slot/multi-band control channel allocation model.

\section{References}

1. M. Pajic, S. Sundaram, G. Pappas, and R. Mangharam, "The wireless control network: A new approach for control over networks," IEEE Transactions on Automatic 
Control, vol. 56, no. 10, pp. 2305-2318, 2011.

2. Y. Mo, T. Kim, K. Brancik, D. Dickinson, H. Lee, A. Perrig, and B. Sinopoli, "Cyber-physical security of a smart grid infrastructure," Proceedings of the IEEE, vol. 100, no. 1, pp. 195-209, 2012.

3. S. Amin, A. Cardenas, and S. Sastry, "Safe and secure networked control systems under denial-of-service attacks," Hybrid Systems: Computation and Control, pp. 31-45, 2009.

4. P. Tague, M. Li, and R. Poovendran, "Mitigation of control channel jamming under node capture attacks," IEEE Transactions on Mobile Computing, vol. 8, no. 9, pp. 1221-1234, 2009.

5. T. Bonaci, P. Lee, L. Bushnell, and R. Poovendran, "A convex optimization approach for clone detection in wireless sensor networks," Pervasive and Mobile Computing, 2012.

6. A. Chan, X. Liu, G. Noubir, and B. Thapa, "Broadcast control channel jamming: Resilience and identification of traitors," IEEE International Symposium on Information Theory, pp. 2496-2500, 2007.

7. L. Lazos, S. Liu, and M. Krunz, "Mitigating control-channel jamming attacks in multi-channel ad hoc networks," Second ACM Conference on Wireless Network Security, pp. 169-180, 2009.

8. T. Bonaci, L. Bushnell, and R. Poovendran, "Node capture attacks in wireless sensor networks: A system theoretic approach," 49th IEEE Conference on Decision and Control (CDC), pp. 6765-6772, 2010.

9. A. Clark, L. Bushnell, and R. Poovendran, "A passivity-based framework for composing attacks on networked control systems," 50th Allerton Conference on Communication, Control, and Computing, 2012.

10. B. Brogliato, O. Egeland, R. Lozano, and B. Maschke, Dissipative Systems Analysis and Control: Theory and Applications. Springer, 2007. 